\title{
The gene encoding the transcriptional regulator Yin Yang 1 (YY1) is a myeloid transforming gene interfering with neutrophilic differentiation
}

Stefan J. Erkeland, Marijke Valkhof, Claudia Heijmans-Antonissen, Ruud Delwel, Peter J. M. Valk, Mirjam H. A. Hermans, and Ivo P. Touw

The genetic defects underlying the pathogenesis of acute myeloid leukemia (AML) are still largely unknown. Retroviral insertion mutagenesis in mice has become a powerful tool to identify candidate genes involved in the development of leukemia and Iymphoma. We have used this strategy with the 1.4 strain of Graffi murine leukemia virus (MuLV), which predominantly causes myeloid leukemias. Here, we report that Graffi-1.4-induced AML frequently harbors virus integrations in the gene encoding the transcription factor Yin Yang 1 (YY1). These integrations occurred in both orientations, and all were located in the $5^{\prime}$ promoter region of the gene, 0.5 to $1.5 \mathrm{~kb}$ upstream of the major transcriptional start site. Luciferase reporter assays showed that virus integration in this region increases promoter activity and renders it independent of a functional binding site for Sp1, a major transcriptional regulator of YY1. We used the murine 32D model to study the consequence of perturbed YY1 expression for myelopoiesis. YY1 protein levels were high in 32D parental cells maintained in interleukin-3-containing medium, but they dropped when the cells were induced to differentiate by granulocyte-colony-stimulating factor (G-CSF). Strikingly, G-CSF-induced neutrophilic differentiation was reduced in 32D cell transfectants ectopically expressing YY1. In similar experiments on primary bone marrow cells, enforced YY1 expression blocked the outgrowth of CFU-GM colonies. Increased YY1 expression was seen in some cases of human AML. Collectively, these data imply a possible role of perturbed expression of YY1 in the development of AML through interference with the myeloid differentiation program in the leukemic progenitor cells. (Blood. 2003; 101:1111-1117)

(c) 2003 by The American Society of Hematology

\section{Introduction}

Human acute myeloid leukemia (AML) is a heterogeneous group of diseases with a variable treatment outcome. It is now well established that responses to different forms of therapy depend to a significant extent on the presence of certain genetic defects in the leukemic cells. For instance, chromosomal abnormalities $t(8 ; 21)$ and $\operatorname{inv}(16) t(15 ; 17)$ are now being used as important prognostic indicators in AML. ${ }^{1}$ However, it has also become clear from in vitro and in vivo models that, when isolated, these defects are insufficient to cause leukemia but that additional "hits" in as yet largely unidentified regulatory genes are required for full leukemic transformation of hematopoietic cells..$^{2,3}$ In addition, in the significant proportion of AML patients who do not have cytogenetic abnormalities, the genes involved in the pathogenesis of the disease remain elusive. Retroviral insertional mutagenesis has become a powerful tool to identify genes implicated in leukemogenesis and lymphomagenesis. ${ }^{2-4}$ In the past, this method has been tedious, but recently developed strategies using inverse polymerase chain reaction (PCR), direct nucleotide sequencing, and database screening now allow for a rapid and large-scale identification of potential disease genes. ${ }^{4}$ Importantly, a number of genes identified by this strategy have also been shown to play a role in human hematopoietic malignancies. ${ }^{4-9}$

Graffi murine leukemia virus (Graffi-MuLV) is an ecotropic retroviral complex causing leukemias in mice. ${ }^{10}$ This viral complex does not contain oncogenic sequences, but it deregulates genes because of proviral integrations. A subclone of this complex, the Graffi-1.4 strain, predominantly induces myeloid leukemias. ${ }^{10}$ In these leukemias, known proto-oncogenes such as c-myc, Pim-1, Fli-1, and Spi-1 are rarely affected, ${ }^{11}$ indicating that other genes involved in Graffi-1.4 MuLV-induced leukemias remain to be discovered.

Yin Yang 1 (YY1, NF-E1, delta, UCRBP, CF1) is a transcription factor of the GLI-Krüppel zinc finger protein family that controls many cellular processes. ${ }^{12-14}$ Targeted disruption of the $Y Y 1$ gene leads to embryonic lethality in mice from severe defects in the development of the embryonic and extraembryonic tissues. ${ }^{15}$ The YY1 gene spans $23 \mathrm{~kb}$ comprising 5 exons and encodes a protein of $44 \mathrm{kDa}$. The gene is located on human chromosome 14q32 and on mouse chromosome 12 and is structurally highly conserved among these species (95\% homology at mRNA level). ${ }^{16}$ Transcription of the $Y Y 1$ gene is tightly controlled. Besides a major transcriptional start site, the existence of 10 alternative start sites in a $(C+G)-$ rich region lacking a TATA box have been reported. ${ }^{17} \mathrm{YY} 1$ expression is controlled by the transcription factor Sp1, which is expressed in many cell types, including hematopoietic cells. ${ }^{17,18}$

YY1 has been reported to activate or repress transcription of a large variety of cellular and viral genes. ${ }^{13,14,19}$ Additionally, YY1 regulates gene expression in a cell-cycle-dependent fashion. This may, at least in part, be due to a control mechanism involving the retinoblastoma protein $(\mathrm{Rb})$, which releases $\mathrm{YY} 1$ in the S-phase of
From the Department of Hematology, Erasmus University Medical Center, Rotterdam, The Netherlands.

Submitted April 22, 2002; accepted September 3, 2002. Prepublished online as Blood First Edition Paper, September 12, 2002; DOI 10.1182/blood-2002-041207.

Supported by grants from the Dutch Cancer Society and the Erasmus University Medical Center (breedtestrategie).
Reprints: Ivo P. Touw, Department of Hematology, Erasmus University Rotterdam, PO Box 1738, 3000 DR Rotterdam, The Netherlands; e-mail: touw@hema.fgg.eur.nl.

The publication costs of this article were defrayed in part by page charge payment. Therefore, and solely to indicate this fact, this article is hereby marked "advertisement" in accordance with 18 U.S.C. section 1734

(C) 2003 by The American Society of Hematology 
the cell cycle..$^{20}$ The transcriptional activity of YY1 is positively regulated through acetylation of the protein by p300 and PCAF and is negatively regulated by deacetylation by histone deacetylases HDAC 1 , HDAC2, and HDAC $3 .^{21}$

In this paper, we report that the YY1 promoter region is a frequent target for integration of Graffi-1.4 MuLV and show that the regulation of YY1 expression is perturbed and becomes independent of $\mathrm{Sp} 1$ regulation as a result of these integrations. We further show that ectopic expression of YY1 has a negative effect on myeloid differentiation in a cell line model and prevents the outgrowth of myeloid progenitors from primary bone marrow cells. Finally, we present data indicating that in certain cases of human AML, YY1 expression is significantly increased compared with healthy bone marrow cells. Based on these findings, we suggest that the deregulation of YY1 expression can contribute to myeloid leukemia by interfering with the normal myeloid differentiation program.

\section{Materials and methods}

\section{Graffi-1.4 MuLV-induced leukemias}

Newborn FVB/N mice (younger than 2 days) were injected subcutaneously with $100 \mu \mathrm{L}$ of a cell culture supernatant of Graffi-1.4 MuLV-producing NIH3T3 cells (a gift from Dr E. Rassart, Departement des Sciences Biologiques, Universite du Quebec a Montreal, Canada). Mice were checked daily for symptoms of illness: apathy, white ears and tail, impaired interaction with cage mates, weight loss, and dull fur. Typically, leukemic mice had enlarged spleens, livers, thymuses, and lymph nodes. From these primary tumors, chromosomal DNA was isolated for PCR-based screening. Blood samples were taken from the heart. For morphologic analysis, blood smears and cytospins were fixed in methanol, stained with May-GrünwaldGiemsa, and analyzed on a Zeiss Axioscope microscope (Carl Zeiss BV, Weesp, The Netherlands).

\section{Immunophenotyping of the leukemic cells}

Single-cell suspensions of different organs were analyzed by flow cytometry using a FACScan flow cytometer (Becton Dickinson, Mountain View, CA). Cells were labeled as described previously ${ }^{22}$ with the following rat monoclonal antibodies: ER-MP54, ER-MP58, M1/70 (Mac-1), F4/80, RB68C5 (GR-1), ER-MP21 (transferrin receptor), TER119 (Glycophorin A), 59-AD2.2 (Thy-1), KT3 (CD3), RA3 6B2 (B220), and E13 1617(Sca1). Immunodetection was performed using a goat anti-rat antibody coupled to fluorescein isothiocyanate (G $\alpha$ Ra-FITC) (Nordic, Tilburg, The Netherlands)

\section{Production of retroviral vectors}

The plasmid pCMV-HA-YY1, containing hemagglutinin (HA)-tagged full-length human YY1 cDNA (a gift from Dr Y. Shi, Department of Pathology, Harvard Medical School, Boston, MA) was digested with Xbal and ApaI, and the HA-YY1 fragment was blunted and ligated into the $\mathrm{Hpal}$ site of pLNCX and pBabe retroviral vectors. ${ }^{23,24}$ PhoenixA packaging cells (from G. Nolan, Stanford, CA) were transfected with pLNCX-HA-YY1 or pBABE-HA-YY1 (Protection Mammalian Transfection Systems E-1200; Promega, Madison, WI). Supernatants containing high-titer, helper-free recombinant virus were harvested from $80 \%$ confluent producer cells cultured for 16 to 20 hours in Dulbecco modified Eagle medium, (DMEM; Gibco-BRL, Breda, The Netherlands) supplemented with 5\% fetal calf serum (FCS), penicillin (100 IU/mL), and streptomycin $(100 \mathrm{ng} / \mathrm{mL})$. To determine titers of BABE-HA-YY1 and BABE control virus, the virus particles were pelleted by ultracentrifugation at $41000 \mathrm{rpm}$ (XL-90; Beckman, Mijdrecht, The Netherlands), and RNA was extracted with phenol $(\mathrm{pH}=4.0)$ and spot-blotted on nitrocellulose filters. This blot was hybridized with a BABE-specific probe (SV40 fragment, BamHIHindIII digest).

\section{Cell culture and retroviral gene transfer}

32D cells. The interleukin-3 (IL-3)-dependent murine myeloid cell line $32 \mathrm{D}^{25}$ containing the human wild-type G-CSF receptor (32D-WT1) were expanded and differentiated as described. ${ }^{26}$ 32D-WT1 cells were infected with pLNCX-HA-YY1 virus and selected with G418 (Gibco-BRL). Several independent clones were expanded for further analysis.

Bone marrow cells. Hematopoietic cells were harvested from the femurs and tibiae of 8 - to 12 -week-old FVB mice as described. ${ }^{27}$ After depletion of adherent cells, the remaining cells were fractionated on a Percoll gradient (AB 17-0891-01; Amersham Pharmacia Biotech, Uppsala, Sweden). Fraction 1 (density 1.058/1.0645) containing the earliest hematopoietic progenitor cells ${ }^{28}$ was collected. Cells were washed twice in Hanks buffered salt solution (HBSS) $/ 5 \%$ FCS/0.5\% bovine serum albumin (BSA) and then were prestimulated for 2 days at a final concentration of $5 \times 10^{5}$ cells/mL in Cell Gro (SCGM BE SP047; Boehringer Ingelheim Bioproducts Partnership Heidelberg, Germany) supplemented with a cytokine cocktail composed of murine (m) IL-3 (10 ng/mL), human (h) Flt3-ligand, human thrombopoietin (hTPO), murine stem cell factor (mSCF; 100 $\mathrm{ng} / \mathrm{mL}$ ) and granulocyte macrophage-colony-stimulating factor (GM-CSF; $2 \mathrm{U} / \mathrm{mL}$ ). Retroviral infection was performed in culture dishes (Falcon 1008; Becton Dickinson) coated with the recombinant fibronectin fragment CH-296 (Takara Shuzo T100A/B; Otsu, Japan) ${ }^{29}$ at a concentration of 12 $\mu \mathrm{g} / \mathrm{mL}$. Before adding the bone marrow cells, the dishes were preincubated with virus supernatant (BABE-HA-YY1 or empty BABE) for 30 minutes at $37^{\circ} \mathrm{C}$. Subsequently, bone marrow cells were resuspended and mixed with fresh virus supernatant in a 1:1 ratio, and a fresh cytokine cocktail was added. Cells $\left(5 \times 10^{5}\right)$ were cultured overnight at $37^{\circ} \mathrm{C}$ and $5 \% \mathrm{CO}_{2}$. Virus supernatant and cytokine cocktail were refreshed again the next day, and cells were cultured for another 24 hours.

Colony assay. Bone marrow cells were plated at densities of 1 to $5 \times 10^{4}$ cells $/ \mathrm{mL}$ per dish in triplicate in methylcellulose medium supplemented with $30 \%$ fetal bovine serum (FBS), $1 \%$ BSA, $0.1 \mathrm{mM} 2$-mercaptoethanol, $2 \mathrm{mM}$ L-glutamine, and GM-CSF (20 U/mL), with or without 2.5 $\mu \mathrm{g} / \mathrm{mL}$ puromycin (Sigma, Zwijndrecht, The Netherlands) to evaluate the infection efficiency of the different retroviruses. Colonies consisting of more than 50 cells were counted on day 7 of culture

\section{Promoter activity assay}

The YY1 promoter-containing subclone p $\delta S S 4.5$ of $\lambda 24^{17}$ was digested with restriction enzymes $M L u \mathrm{I}$ and $B g I I I$. This YY1 promoter fragment was cloned in the luciferase reporter plasmid pGL3 (Promega). The Graffi-1.4 MuLV long terminal repeat (LTR) sequence was cloned in both orientations in the HpaI site. The Sp1 site was mutated with the QuikChange Site-Directed Mutagenesis Kit (Stratagene, La Jolla, CA) by using primer SPIMUTF (5' GGACGGTTCGGGGCGAGAGC $3^{\prime}$ ) and primer SPIMUTR (5' GCTCTCGCCCCGAACCGTCC 3'). As a positive control, pRSV$\mathrm{Luc}^{30}$ was used. pRSV- $\beta$-galactosidase (a gift from C. Berrevoets, Erasmus University, Rotterdam, The Netherlands) was used for reference. Empty pGL3 vector served as a negative control. Luciferase assays were performed in HEK 293 cells. Cells were transfected by $\mathrm{CaPO}_{4}$ precipitation ${ }^{31}$ with a mixture of $5 \mu \mathrm{g}$ derived pGL3 plasmid containing different promoter sequences and $2.5 \mu \mathrm{g}$ pRSV- $\beta$-galactosidase, supplemented with empty vector, to a total amount of $20 \mu \mathrm{g}$ total plasmid per milliliter. Luciferase assays were performed as described, ${ }^{26}$ and activities were calculated in arbitrary units, relative to $\beta$-galactosidase expression.

Twenty-five microliters cell lysate was mixed with $75 \mu \mathrm{L}$ ONPG solution (0.028 g 2-nitro-phenyl-galacto-pyranosid; Boehringer-Mannheim, Almere, The Netherlands), $50 \mathrm{~mL}$ P-buffer (100 mM phosphate buffer, $\mathrm{pH} 7.0$ ), $10 \mathrm{mM} \mathrm{MgSO}{ }_{4}, 2.7 \mathrm{mM}$ ). Samples were incubated at $37^{\circ} \mathrm{C}$ for 1 hour. The extinction was measured at $450 \mathrm{~nm}$ in a Bio-Rad 450 microplate reader (Bio-Rad, Richmond, CA). 


\section{Inverse PCR on Graffi-1.4 MuLV-induced leukemias}

Genomic DNA from the primary tumors was digested with HhaI. After ligation (Rapid ligation kit; Roche Diagnostics, Mannheim, Germany), a first PCR was performed using Graffi-1.4 MuLV (LTR) specific primers L1 (5' TGCAAGATGGCGTTACTGTAGCTAG $3^{\prime}$ ) and L2 (5' CCAGGTTGCCCCAAAGACCTG $3^{\prime}$ ) (cycling conditions were 1 minute at $94^{\circ} \mathrm{C}, 1$ minute at $65^{\circ} \mathrm{C}$, and 3 minutes at $72^{\circ} \mathrm{C} ; 30$ cycles). For the second nested PCR, the primers L1N ( $5^{\prime}$ AGCCTTATGGTGGGGTCTTTC $\left.3^{\prime}\right)$ and L2N (5' AAAGACCTGAAACGACCTTGC 3') (15 cycles) were used. The PCR reaction mixture contained $10 \mathrm{mM}$ Tris(tris[hydroxymethyl]aminomethane)$\mathrm{HCl}(\mathrm{pH}$ 8.3), $50 \mathrm{mM} \mathrm{KCl}, 1.5 \mathrm{mM} \mathrm{MgCl} 2,200 \mu \mathrm{M}$ dNTP, 10 pmol each primer, and $2.5 \mathrm{U}$ Taq polymerase (Pharmacia, Uppsala, Sweden). PCR fragments were analyzed on a $1 \%$ agarose gel and cloned in the TA cloning vector (Invitrogen BV, Carlsbad, CA) according to standard procedures.

\section{Detection of virus integration in the $Y Y 1$ gene by specific nested PCR}

To determine the orientation and localization of the Graffi-1.4 provirus in the $Y Y 1$ gene in an extended panel of leukemias, nested PCR was performed on $1 \mu \mathrm{g}$ genomic DNA from primary tumors. For the first PCR, YY1 promoter region-specific primers Y1 (5' AGGAATCAGGAGCAGAAGAAAGTTTTGGGA 3') and Y2 (5' CAATAAAGTCTGCTCTGACGAGAAACGCC $3^{\prime}$ ), in combination with Graffi-1.4 MuLV LTR-specific primers L1 and L2 were used. Cycling conditions were 1 minute at $94^{\circ} \mathrm{C}, 1$ minute at $65^{\circ} \mathrm{C}$, and 3 minutes at $72^{\circ} \mathrm{C}$, for 30 cycles. For the second PCR, the nested primers Y1N (5' AAACTCTCTGACTTACCTCCCTCTCCAAAGA $\left.3^{\prime}\right)$ and Y2N (5' GTTCGTTTTGCCTTTACTCGTTACTCGGG $3^{\prime}$ ), in combination with the nested primers L1N and L2N (30 cycles), were used. The obtained PCR products were analyzed by Southern blotting. To determine the orientation of the Graffi-1.4 provirus, the blots were hybridized with radiolabeled Y1P (5' AAAACCTGCACAAGGACACCTTGCTAAGTATGTTT $\left.3^{\prime}\right)$ and Y2P (5' AGCACACGGTCGGCTACGCTCCGTCCGCTACCGCA $\left.3^{\prime}\right)$ at $45^{\circ} \mathrm{C}$ in Church buffer (0.5 M phosphate buffer, $\mathrm{pH}$ 7.2, 7\% [wt/vol] sodium dodecyl sulfate [SDS], $10 \mathrm{mM}$ EDTA [ethylenediaminetetraacetic acid]) overnight. Signals were visualized by autoradiography according to standard procedures.

\section{Nucleotide sequencing}

PCR products were cloned in TA cloning vector and sequenced with the M13 forward primer (5' GACCGGCAGCAAAATG 3') and M13 reverse primer (5' CAGGAAACAGCTATGAC 3') using an ABI 3100 sequencer (Perkin Elmer, Nieuwerkerk a/d IJssel, The Netherlands). Virus-flanking genomic sequences were identified using the National Center for Biotechnology Information (NCBI) database.

\section{Western blotting}

Lysates of 32D cells were prepared and subjected to Western blotting as described previously. ${ }^{32}$ Antibodies used to visualize YY1 were goat anti-YY1 (Santa Cruz Biotechnology, CA) or rabbit anti-HA (Y-11, sc-805) for HA-tagged YY1. Rabbit anti-Sp1 (a gift from Dr G. Suske, PhilippsUniversity, Marburg, Germany) was used for the detection of Sp1. Goat anti-actin (I-19, sc-1616; Santa Cruz Biotechnology) was used to control for equal loading of lysates.

\section{Real-time quantitative PCR on human AML samples}

Human AML cells were obtained following informed consent and were purified as previously described. ${ }^{33}$ These purified blast fractions consisted of more than $95 \%$ myeloblasts. Total RNA was extracted with guanidinium thiocyanate and was purified by cesium chloride gradient centrifugation. RNA was transcribed into cDNA under standard conditions using Superscript (Life Technologies, Merelbeke, Belgium) and random hexamers. Real-time PCR amplification (ABI PRISM 7700 Sequence Detector; PE Biosystems, Nieuwerkerk a/d IJssel, The Netherlands) was performed in a mix of $50 \mu \mathrm{L}$ containing $2 \mu \mathrm{L}$ cDNA, $250 \mu \mathrm{M}$ dNTP (Amersham Pharmacia Biotech, Roosendaal, The Netherlands), $3 \mathrm{mM} \mathrm{MgCl}_{2}, 15 \mathrm{pmol}$ primers, $200 \mathrm{nM}$ Taqman probe, labeled $5^{\prime}$ with reporter dye FAM (6-carboxy-fluorescein) and $3^{\prime}$ with quencher dye TAMRA (6-carboxytetramethyl-rhodamine; Eurogentec, Maastricht, The Netherlands), $1.25 \mathrm{U}$ AmpliTaq Gold (PE Applied Biosystems), $5 \mu \mathrm{L} 10 \times$ buffer A (PE Applied Biosystems), and $30 \mu \mathrm{L} \mathrm{H} \mathrm{H}_{2} \mathrm{O}$. Cycling conditions were 2 minutes at $50^{\circ} \mathrm{C}$ and 10 minutes at $95^{\circ} \mathrm{C}$, followed by 45 cycles of denaturation ( 15 seconds at $95^{\circ} \mathrm{C}$ ), and annealing/extension $\left(1\right.$ minute at $\left.60^{\circ} \mathrm{C}\right)$. For YY1, forward primer (5' ATACCTGGCATTGACCT $3^{\prime}$ ) and reverse primer (5' TGAGGGCAAGCTATTGT 3') were used. The YY1 Taqman probe was 5' GAATGAAGCCAAGAAAAATTAAAGAAGATGT $3{ }^{\prime}$. The housekeeping gene porphobilinogen deaminase $(P B G D)$ was taken as endogenous reference ( $P B G D$ forward primer 5' GGCAATGCGGCTGCAG 3'; $P B G D$ reverse primer 5' GGGTACCCACGCGAATCAC 3'; $P B G D$ Taqman probe 5' CATCTTTGGGCTGTTTTCTTCCGCC 3'). All samples were tested in duplicate, and average values were used for quantification. The expression of YY1 in patient samples relative to YY1 expression in healthy bone marrow $(n=6)$ was calculated according to manufacturer's instructions (user bulletin 2; ABI PRISM 7700 Sequence Detector; PE Biosystems).

\section{Results}

\section{Graffi-1.4 MuLV-induced leukemias}

Leukemias developed 4 to 6 months after subcutaneous injection of newborn FVB/N mice with Graffi-1.4 MuLV. Forty-eight of 59 $(81 \%)$ leukemias analyzed exhibited morphologic characteristics of myeloid cells. Blast cell percentages in the bone marrow ranged from $24 \%$ to $90 \%$, with an average of $48 \%$. Leukemia cells expressed immunophenotypic marker profiles consistent with their

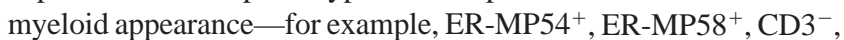
GR $-1^{+}$. Six leukemias with blastlike morphology showed no immunophenotypic differentiation markers, suggesting that these tumors represented immature leukemias. Only 3 leukemias were of T-lymphoid origin $\left(\mathrm{CD}^{+} / \mathrm{MP}^{-} 8^{-} / \mathrm{Thy}^{+}\right)$, and 2 showed mixed myeloid and erythroid features (Ter $\left.119^{+} / \mathrm{ER}-\mathrm{MP} 58^{+} / \mathrm{F} 4 / 80^{+}\right)$. These results demonstrate that Graffi-1.4 MuLV infection predominantly induces myeloid leukemia in FVB/N mice.

\section{Virus integrations in the $Y Y 1$ gene}

Inverse PCR was used to identify genomic sequences flanking Graffi1.4 MuLV integrations (Figure 1A). Integrations were found in several genes previously demonstrated to be involved in MuLV-induced leukemias (eg, Notch-1, Nf1, p53, Fli-1, Evi-1) and in several novel loci (S.J.E. et al, manuscript in preparation). One of these newly identified integrations occurred in the YY1 locus, approximately $0.8 \mathrm{~kb}$ upstream of the transcriptional initiation site. In a subsequent analysis on independent cell samples using nested PCR with LTR and YY1 primers (Figure 1B-C), we found 23 integrations in the same region, 0.5 to $1.5 \mathrm{~kb}$ upstream of the transcriptional start site, in 14 of 20 leukemias tested. Integrations occurred in both orientations at an approximately equal ratio. Representative examples of these integrations are shown in Figure 1D. Searches for virus integrations in other parts of the gene using appropriate primer sets were negative (data not shown). It is of note that in some leukemia samples multiple integrations were detected, indicating that the Graffi-1.4 MuLV-induced leukemias are oligoclonal.

\section{Integration of Graffi-1.4 MuLV LTR deregulates YY1 transcription}

Because integration of the virus occurred in both orientations, we considered it most likely that viral enhancer sequences in the LTR 
A
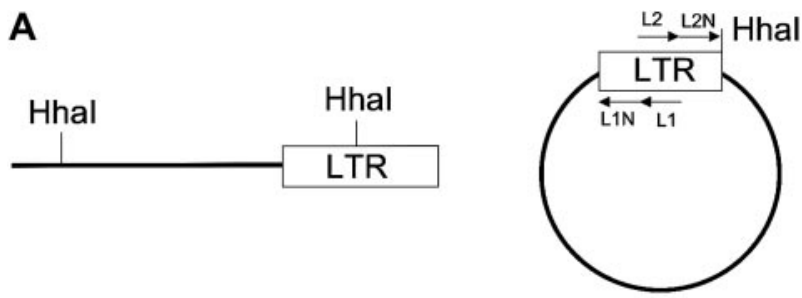

B

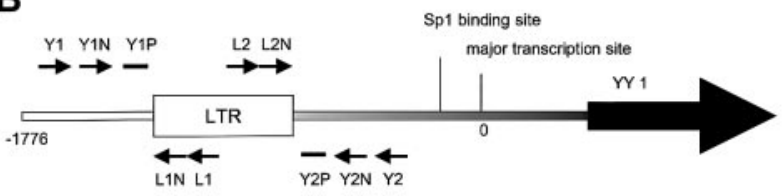

C

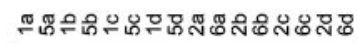

D

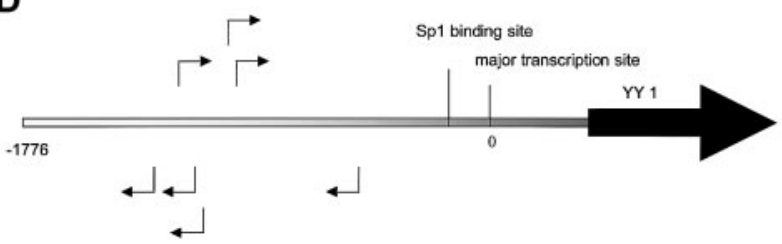

Figure 1. Identification of common virus integrations in the YY1 locus. (A) Inverse PCR. Genomic DNA from leukemia cells was digested with Hhal. After ligation PCR was performed with primers L1 and L2 followed by a nested PCR with primers L1N and L2N to amplify LTR-flanking fragments from circularized DNA (B) Nested PCR with LTR and YY1 primers to detect position and orientation of Graffi-1.4 MuLV integration in the YY1 promoter region. The lowercase letters indicate the position at the promoter in base pairs (bp). The first PCR was performed with the primer sets L1, Y1 (a), L1, Y2 (b), L2, Y1 (c), and L2, Y2 (d), followed by a nested PCR with primers L1N, Y1N (a) and L1N, Y2N (b), L2N, Y1N (c), and L2N Y2N (d). Probes Y1P and Y2P were used to analyze the specificity of the PCR band by Southern blot. (C) Example of Southern blot analysis to determine virus integration and orientation in the $5^{\prime}$ region of the $Y Y 1$ gene. Results depicted are from DNA samples of leukemias $1,2,5$, and 6 . PCR products from the 4 different primer combinations ( $\mathrm{a}, \mathrm{b}, \mathrm{c}$, and d) were analyzed. In this example, the blot was hybridized with probe Y1P. The presence of the band in lane $1 \mathrm{c}$ indicates that tumor 1 has a virus integration in the reverse orientation. Tumor 5 has 2 YY1 virus integrations in the reverse orientation (lane $5 \mathrm{c}$ ). Tumor 2 has an integration in the forward orientation (lane 2a). Tumor 6 harbors 2 integrations in both orientations (lanes $6 \mathrm{a}$ and $6 \mathrm{c}$ ). Al bands were sequenced to determine the exact location of virus integration. (D) Examples of virus integrations and orientations found in the YY1 promoter.

cause increased YY1 transcription in these types of leukemia. Integration in the promoter region of a gene, however, does not always result in alterations in expression. For instance, the common ecotropic virus integration 12 (Evi12) is located approximately $1 \mathrm{~kb}$ upstream of the transcriptional start site of the Tral/Grp94 gene but does not modulate its expression. ${ }^{34}$ We first compared YY1 expression in the murine leukemias with and without virus integration by Northern and Western blot analyses but found no major differences between the groups (data not shown). However, because virally induced leukemias are oligoclonal $^{2,4}$ (and S.J.E. et al, unpublished data), abnormalities in

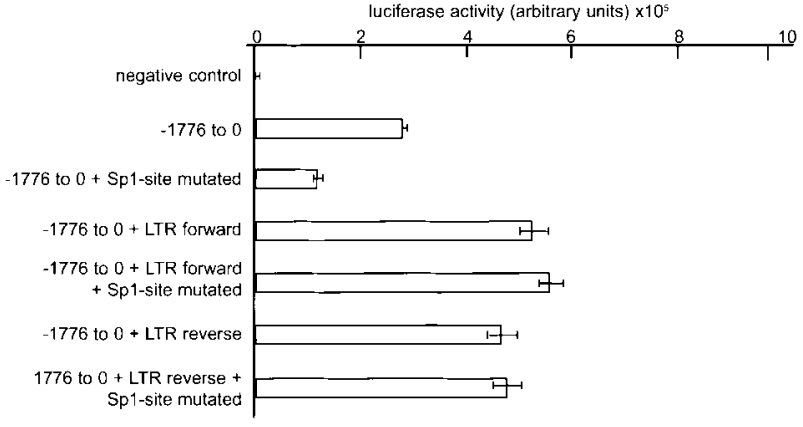

Figure 2. Effects of Graffi-1.4 LTR integration in the YY1 promoter region on gene transcription. Luciferase assays were performed in HEK 293 cells. One representative experiment of 3 is shown. Error bars represent the SD of the mean values of 3 independent experiments. Negative control: empty pGL3 vector. Reporter gene expression was calculated in arbitrary units, relative to $\beta$-galactosidase expression.

expression of YY1 in a subset of leukemia cells might go undetected by these techniques. As an alternative approach, we introduced the Graffi-1.4 LTR in the Hpa1 site at position - 1417 in the YY1 promoter and quantified transcriptional activity using a transient reporter assay (Figure 2). Twofold higher YY1 promoter activity was measured following insertion of the Graffi-1.4 MuLV LTR, irrespective of the orientation of the LTR (Figure 2). Because an Sp1-binding site at position -48 to -39 has previously been shown to be important for the induction of YY1 transcription, ${ }^{17}$ we also studied the effects of insertion of the Graffi-1.4 MuLV LTR after disruption of this site. Although mutation of the Sp1-binding site resulted in a $50 \%$ reduction of normal promoter activity, the enhanced promoter activity caused by the integration of the LTR sequence was not affected (Figure 2). This result demonstrates that virus integration causes enhanced and $\mathrm{Sp} 1$-independent transcription of YY1.

\section{Ectopic expression of YY1 blocks G-CSF-induced neutrophilic differentiation of 32D cells}

The observation that integration of Graffi MuLV LTR alters the expression of YY1 in murine acute myeloid leukemia raised the question whether perturbed YY1 expression might affect myeloid cell differentiation. We have previously developed 32D cell clones expressing human G-CSF receptors (32D-WT1 cells) as a model to study neutrophilic differentiation. ${ }^{26}$ Endogenous YY1 levels are high in parental 32D-WT1 cells cultured in IL-3-containing medium, under which conditions the cells remain immature (Figure 3). However, on transfer of the cells to a G-CSF-containing medium, in which cells undergo neutrophilic differentiation, YY1 protein levels declined from day 2 onward and remained low for the entire culture period (Figure 3 ). In view of the regulatory role of

\section{IL-3 G-CSF (days in culture)}

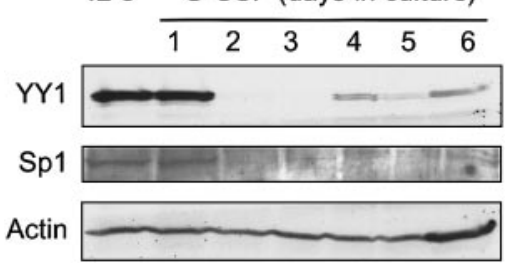

Figure 3. Western blot analysis of YY1 expression in 32D-WT1 cells. G-CSF responsive 32D-WT1 cells were cultured in IL-3-containing medium and then switched to G-CSF. Samples were taken daily and processed as described in "Materials and methods." The blot was hybridized with anti-YY1 or anti-Sp1, stripped, and rehybridized with anti-Actin to check for equal loading of cell lysates. 
A

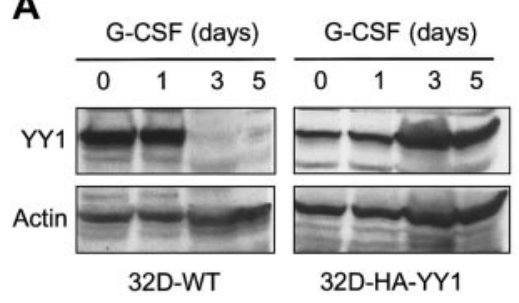

B
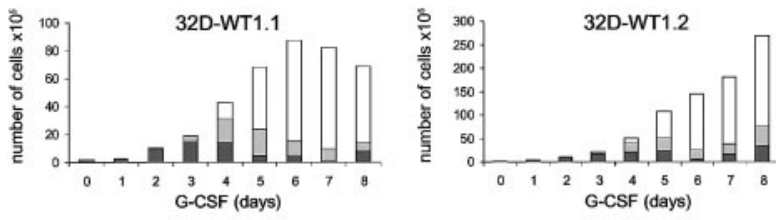

32D-HA-YY1.1

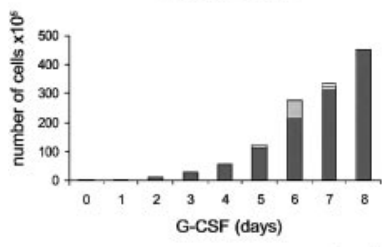

○ granulocyte

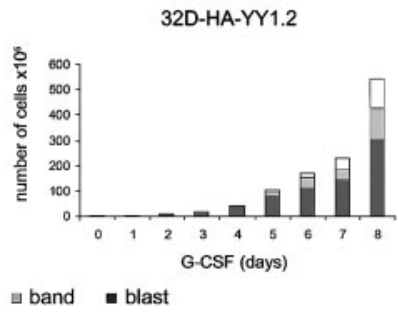

C

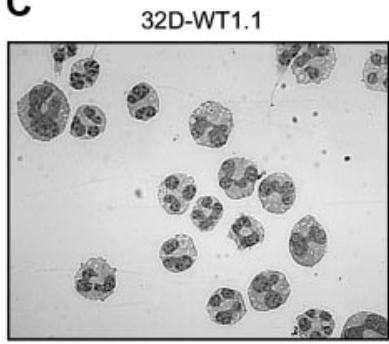

32D-HA-YY1.1
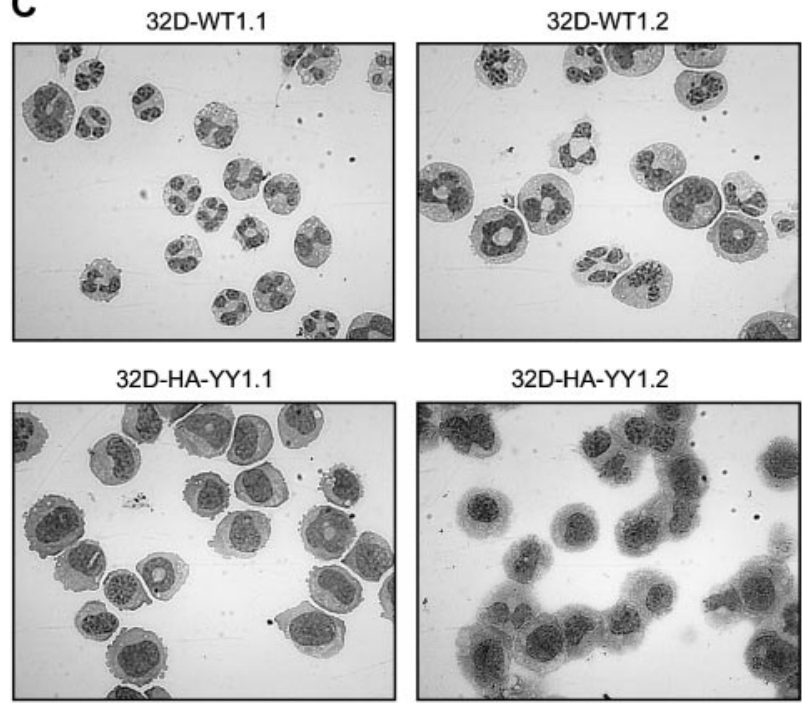

32D-HA-YY1.2

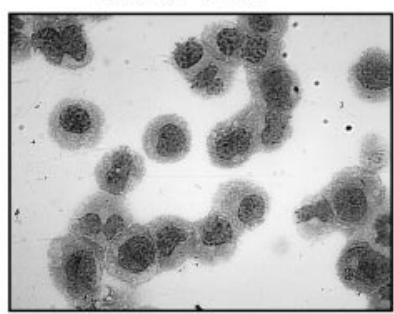

Figure 4. Ectopic expression of HA-YY1 in 32D cells inhibits neutrophilic differentiation. (A) Western blot analysis with anti-YY1 in 32D-WT1 and with anti-HA antibodies in 32D-HA-YY1 cells after switching the cells from IL-3- to G-CSFcontaining medium on $t=0$ days. The blot was reprobed with anti-actin antibodies for loading control. (B) Differential cell count (blasts, band form, and segmented nuclei) of 2 representative 32D-WT1 and 2 representative 32D-HA-YY1 clones. (C) Micrographs showing morphology of 32D-WT1 and 32D-HA-YY1 clones on day 5 (original magnification, $\times 1000$ ).

Sp1 in YY1 expression, we also looked at Sp1 levels and found that expression of Sp1 was down-regulated in the same time frame after switching the cells to G-CSF. These results raise the possibility that YY1 levels were reduced as a consequence of lowered Sp1controlled transcription. To determine the consequences of perturbed YY1 expression for myeloid cell development, we ectopically expressed HA-tagged YY1 in these cells. As expected, YY1 expression in 32D-HA-YY1 cells remained high after the cells were switched to G-CSF (Figure 4A). Growth rate and morphology of 32D-HA-YY1 cells cultured in IL-3-containing medium were similar to nontransduced control cells (data not shown). Strikingly, when 32D-HA-YY1 cells were switched to G-CSF-containing medium, neutrophilic differentiation was markedly reduced (Figure 4B-C). Instead, cells with a blastlike morphology expanded exponentially for 8 days in these cultures (Figure 4B) and rapidly died thereafter as a result of apoptosis (data not shown). Thus, sustained YY1 expression prevented G-CSF-induced myeloid differentiation of 32D cells without affecting the apoptotic response that normally accompanies G-CSF-induced differentiation.

\section{Ectopic expression of YY1 blocks CFU-GM colony formation}

The above-mentioned experiments indicated that constitutive expression of YY1 interferes with G-CSF-induced myeloid differentiation in 32D cells. We next wanted to investigate how perturbed expression of YY1 affects the outgrowth of primary myelomonocytic progenitor cells (granulocyte macrophage-colony-forming unit $[\mathrm{CFU}-\mathrm{GM}])$. To this end, we retrovirally transduced mouse bone marrow cells with BABE-HA-YY1 or empty BABE virus and plated these in GM-CSF-containing colony assays. Notably, control bone marrow cells were subjected to equivalent titers of control virus (Figure 5A) to exclude that differences in colony outgrowth were caused by variations in transduction efficiencies. Strikingly, GM-CSF-induced colony formation by HA-YY1-transduced bone marrow cells was almost completely blocked (Figure 5B). This result suggests that perturbed expression of YY1, instead of interfering with differentiation, causes a growth arrest or results in premature apoptosis of CFU-GMs or their direct progeny.

A $\mu \mathrm{L}$ supernatant

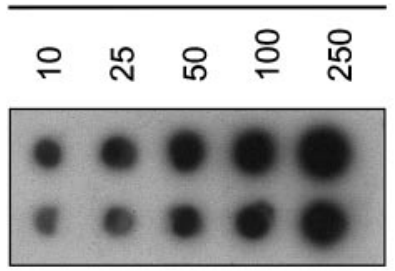

BABE-HA-YY1

BABE

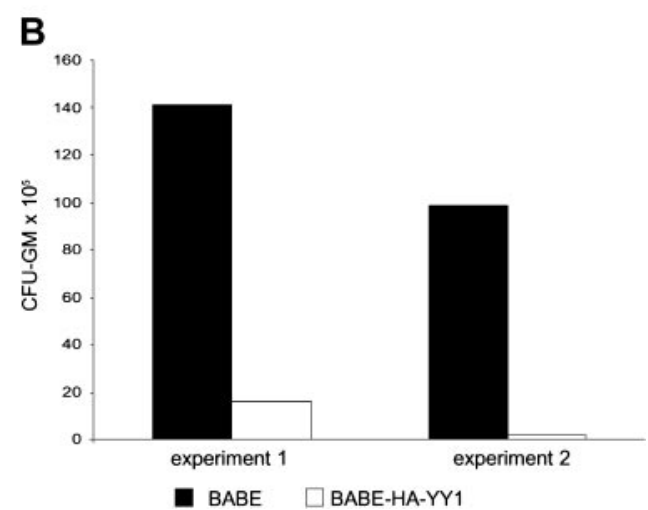

Figure 5. GM-CSF-induced colony formation by primary bone marrow cells after retroviral transduction of YY1. (A) RNA spot blot analysis of supernatants containing $\mathrm{BABE} / \mathrm{HA}-\mathrm{YY} 1$ or $\mathrm{BABE}$ vector control virus, showing that titers used for infection were comparable. The filter was hybridized with a BABE-specific cDNA probe. (B) GM-CFU assay of primary bone marrow progenitor cells following infection with $\mathrm{BABE}-\mathrm{HA}-\mathrm{YY} 1$ or BABE control virus. Bone marrow cells were plated in triplicate at densities of 10 to $50 \times 10^{3}$ cells per dish in $1 \mathrm{~mL}$ methylcellulose medium containing GM-CSF $(20 \mathrm{U} / \mathrm{mL})$ and puromycin $(2.5 \mu \mathrm{g} / \mathrm{mL})$. Two independent experiments are shown. 


\section{YY1 expression in human AML}

An important question is whether increased levels of YY1 expression can also be found in human AML cells, which would be suggestive of a possible involvement of abnormal YY1 expression in human disease. We performed real-time quantitative PCR on cDNAs from 94 patients with AML and 6 healthy volunteers. As shown in Figure 6, YY1 transcript levels in most patients did not significantly differ from healthy bone marrow cells. However, in 13 patients, mRNA levels significantly exceeded those of the healthy bone marrow controls.

\section{Discussion}

In this study, we demonstrated that the gene encoding the transcriptional regulator YY1 is located in a new common virus integration site in Graffi-1.4 MuLV-induced myeloid leukemia. The integrations occurred exclusively in the $5^{\prime}$ promoter region of the gene, 0.5 to $1.5 \mathrm{~kb}$ upstream of the major transcriptional start site. Data from luciferase reporter assays strongly suggested that these integrations result in the dysregulation of YY1 expression. The pattern of virus integrations indicated that Graffi-1.4 MuLVinduced leukemias are oligoclonal, which is consistent with observations on leukemias induced by other MuLVs ${ }^{2,4}$ (and S.J.E. et al, unpublished data). The $Y Y 1$ gene has already been identified once by inverse PCR as a site of retroviral integration in $\mathrm{BXH} 2$ leukemia/lymphoma. ${ }^{4}$ Although the exact site of integration and the type of leukemia/lymphoma were not specified in this study, the finding that the YY1 locus is affected by distinct MuLV types in different mouse strains indicates that the deregulation of YY1 may be more common in the development of various hematologic malignancies in mice.

Perturbed expression of YY1 inhibited G-CSF-induced myeloid differentiation in 32D cells and prevented the clonal outgrowth of CFU-GM progenitor cells. Importantly, we observed that integration of Graffi-virus LTR sequences enhanced YY1 transcription and made it entirely independent of the recognition site for the transcription factor $\mathrm{Sp} 1$, which has been shown to play a pivotal role in YY1 expression. Although Sp1 expression has been reported to be high in hematopoietic cells, it is down-regulated during differentiation in many cell types. ${ }^{18}$ Indeed, we found that Sp1 declined in 32D cells after 2 days of G-CSF-induced differentiation (Figure 3). Therefore, we suggest that the bypass of Sp1 transcriptional control might be one of the mechanisms by which viral integration deregulates YY1 protein levels in leukemic cells.

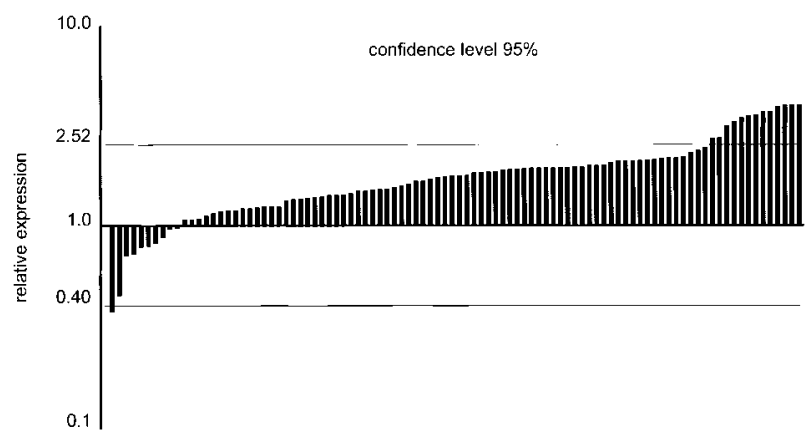

Figure 6. Real-time quantitative PCR analysis of YY1 transcripts in 94 patients with AML. Data are relative to the mean expression in healthy bone marrow samples $(n=6)$, with $95 \%$ confidence limits indicated by the horizontal lines. AML data represent the mean of 2 independent experiments. The $95 \%$ confidence interval was calculated as: $X_{\text {mean }}(6$ NBM samples) $\pm(1.96 \times \mathrm{SD})$.
To establish whether down-regulation of YY1 resulted from G-CSF-induced differentiation rather than from simply switching the cells from IL-3- to G-CSF-containing medium, we also analyzed YY1 expression in IL-3-dependent Ba/F3 transfectants expressing the G-CSF-R. These cells proliferate, but they do not undergo differentiation in response to G-CSF. Because YY1 levels remained high in $\mathrm{Ba} / \mathrm{F} 3-\mathrm{G}-\mathrm{CSF}-\mathrm{R}$ cells (data not shown) cultured in G-CSF-containing medium, it appears most likely that YY1 expression is reduced during G-CSF-induced differentiation. This notion is further supported by observations in 32D cells expressing a truncated G-CSF receptor, ${ }^{35}$ which fails to differentiate in response to G-CSF and retains YY1 expression at a level comparable to that of cells cultured in IL-3 (data not shown)

A major question that remains to be addressed is how deregulation of YY1 expression leads to the observed defects in myeloid cell development. In view of the myriad proposed functions of YY1, multiple possibilities can be envisaged that are not mutually exclusive. First, it is conceivable that constitutive YY1 expression directly influences the transcriptional regulation of genes controlling myeloid cell development. This might be due either to the action of YY1 as a positive regulator of transcription or to its repressor activity. With regard to the latter, it is of interest that YY1 interacts with the EED-EZH (enhancer of Zeste) polycomb complex $(\mathrm{PcG})$ involved in transcriptional repression. In this context, YY1 plays a role in targeting the PcG complex to specific DNA sequences and recruiting HDACs, which associate with both YY1 and EED. ${ }^{36,37}$ It is conceivable that the deregulation of YY1 could cause sustained repression of multiple target genes critical for granulocytic differentiation. In fact, altered recruitment of HDACs has already been implicated in the deregulation of myeloid differentiation by a number of transcription factor fusion proteins generated by specific translocations-PLZF-RAR $\alpha \mathrm{t}(11 ; 17),{ }^{38} \mathrm{PML}-$ RAR $\alpha \mathrm{t}(15 ; 17),{ }^{39}$ and AML1-ETO $\mathrm{t}(8 ; 21) .{ }^{40,41}$

An alternative mechanism by which YY1 can influence cell fate is by forming complexes with other protein regulators, including the $\mathrm{c}-\mathrm{Myc}$ and retinoblastoma $(\mathrm{Rb})$ proteins. ${ }^{20,42,43} \mathrm{~A}$ previous study has demonstrated that $\mathrm{YY} 1$ protein binds to $\mathrm{Rb}$ in a cell-cycle-dependent manner. ${ }^{20}$ In the $\mathrm{G}_{0} / \mathrm{G}_{1}$ phase of the cell cycle, YY1 is in a complex with Rb and cannot bind DNA but is released from this complex when cells enter into S-phase, thereby regaining its DNA-binding activity. ${ }^{20}$ The $\mathrm{Rb}$ protein family comprises 3 members, Rb, p107, and p130. Rb and p107, but not p130, are abundantly present in 32D cells proliferating in IL-3containing medium. Conversely, on G-CSF-induced differentiation, $\mathrm{Rb}$ and p107 levels declined as p130 protein levels dramatically increased, suggesting that p130 plays a role in the induction of myeloid differentiation. ${ }^{44}$ Based on these findings, we hypothesized that the overexpression of YY1 might result in sustained binding to p130 and thereby might interfere with differentiation. Although we confirmed that YY1 can form a complex with Rb in coimmunoprecipitation assays, we have been unable to detect such an interaction of YY1 with the p130 or p107 proteins (data not shown).

An important extension of this work is to determine whether abnormalities in YY1 expression may also contribute to the pathogenesis of human hematopoietic malignancies. In a first attempt to address this question, we performed real-time quantitative PCR on cDNAs from purified human AML blasts and observed that in 13 patients, the expression of YY1 was significantly elevated compared with that in healthy bone marrow controls. Although this would argue in favor of the possibility that YY1 transcript levels are abnormal in these patients, accurate 
comparisons are difficult because healthy bone marrow contains a mixture of cell types of different lineages in various stages of maturation. Identification of the genes controlled by YY1 during myelopoiesis and a more detailed understanding of the signaling pathways influenced by YY1 in hematopoietic cells will be required to shed light on how perturbations in these processes contribute to the development of human AML.

\section{Acknowledgments}

We thank Dr E. Rassart for the Graffi-1.4 MuLV. We also thank Drs Joanna Prasher, Bart Aarts, and Marieke von Lindern for their comments on this manuscript and Karola van Rooyen for assistance with the preparation of figures.

\section{References}

1. Lowenberg B, Downing JR, Burnett A. Acute myeloid leukemia. N Engl J Med. 1999;341:10511062

2. Jonkers J, Berns A. Retroviral insertional mutagenesis as a strategy to identify cancer genes. Biochim Biophys Acta. 1996;1287:29-57.

3. Largaespada DA. Genetic heterogeneity in acute myeloid leukemia: maximizing information flow from MuLV mutagenesis studies. Leukemia. 2000;14:1174-1184.

4. Li J, Shen H, Himmel KL, et al. Leukaemia disease genes: large-scale cloning and pathway predictions. Nat Genet. 1999;23:348-353.

5. Look AT. Oncogenic transcription factors in the human acute leukemias. Science. 1997;278: 1059-1064.

6. Thorsteinsdottir $U$, Kroon E, Jerome L, Blasi F, Sauvageau G. Defining roles for HOX and MEIS1 genes in induction of acute myeloid leukemia. Mol Cell Biol. 2001;21:224-234.

7. Fujino T, Yamazaki Y, Largaespada DA, et al. Inhibition of myeloid differentiation by Hoxa9, Hoxb8, and Meis homeobox genes. Exp Hematol. 2001;29:856-863.

8. Nucifora G. The EVI1 gene in myeloid leukemia. Leukemia. 1997;11:2022-2031.

9. Bruckert $\mathrm{P}$, Kappler R, Scherthan $\mathrm{H}$, et al. Double minutes and c-MYC amplification in acute myelogenous leukemia: are they prognostic factors? Cancer Genet Cytogenet. 2000;120:73-79.

10. Ru M, Shustik C, Rassart E. Graffi murine leukemia virus: molecular cloning and characterization of the myeloid leukemia-inducing agent. J Virol. 1993;67:4722-4731.

11. Denicourt C, Edouard E, Rassart E. Oncogene activation in myeloid leukemias by Graffi murine leukemia virus proviral integration. J Virol. 1999; 73:4439-4442

12. Austen M, Luscher B, Luscher-Firzlaff JM. Characterization of the transcriptional regulator $Y Y 1$ : the bipartite transactivation domain is independent of interaction with the TATA box-binding protein, transcription factor IIB, TAFII55, or CAMP-responsive element-binding protein (CPB)binding protein. J Biol Chem. 1997;272:17091717.

13. Shi Y, Lee JS, Galvin KM. Everything you have ever wanted to know about Yin Yang 1. Biochim Biophys Acta. 1997;1332:F49-F66.

14. Thomas MJ, Seto E. Unlocking the mechanisms of transcription factor YY1: are chromatin modify ing enzymes the key? Gene. 1999;236:197-208.

15. Donohoe ME, Zhang X, McGinnis L, et al. Targeted disruption of mouse Yin Yang 1 transcription factor results in peri-implantation lethality. Mol Cell Biol. 1999;19:7237-7244.

16. Yao YL, Dupont BR, Ghosh S, et al. Cloning, chromosomal localization and promoter analysis of the human transcription factor YY1. Nucleic Acids Res. 1998;26:3776-3783.

17. Safrany G, Perry RP. Characterization of the mouse gene that encodes the delta/YY1/NF-E1/
UCRBP transcription factor. Proc Natl Acad Sci U S A. 1993;90:5559-5563.

18. Saffer JD, Jackson SP, Annarella MB. Developmental expression of Sp1 in the mouse. Mol Cell Biol. 1991;11:2189-2199.

19. Shrivastava A, Calame K. An analysis of genes regulated by the multi-functional transcriptional regulator Yin Yang-1. Nucleic Acids Res. 1994;22: 5151-5155.

20. Petkova V, Romanowski MJ, Sulijoadikusumo I, et al. Interaction between YY1 and the retinoblastoma protein: regulation of cell cycle progression in differentiated cells. J Biol Chem. 2000;276: 7932-7936.

21. Yao YL, Yang WM, Seto E. Regulation of transcription factor $Y Y 1$ by acetylation and deacetylation. Mol Cell Biol. 2001;21:5979-5991.

22. Joosten M, Valk PJ, Vankan Y, et al. Phenotyping of Evi1, Evi11/Cb2, and Evi12 transformed leukemias isolated from a novel panel of cas-Br-M murine leukemia virus-infected mice. Virology. 2000; 268:308-318.

23. Miller AD, Rosman GJ. Improved retroviral vectors for gene transfer and expression. Biotechniques. 1989;7:980-986, 989.

24. Morgenstern JP, Land H. Advanced mammalian gene transfer: high titre retroviral vectors with multiple drug selection markers and a complementary helper-free packaging cell line. Nucleic Acids Res. 1990;18:3587-3596.

25. Greenberger JS, Sakakeeny MA, Humphries RK Eaves CJ, Eckner RJ. Demonstration of permanent factor-dependent multipotential (erythroid/ neutrophil/basophil) hematopoietic progenitor cell lines. Proc Natl Acad Sci U S A. 1983;80:29312935.

26. de Koning JP, Soede-Bobok AA, Ward AC, et al. STAT3-mediated differentiation and survival and of myeloid cells in response to granulocyte colony-stimulating factor: role for the cyclindependent kinase inhibitor p27(Kip1). Oncogene. 2000;19:3290-3298

27. Hermans $\mathrm{MH}$, Ward AC, Antonissen $\mathrm{C}$, et al. Perturbed granulopoiesis in mice with a targeted mutation in the granulocyte colony-stimulating factor receptor gene associated with severe chronic neutropenia. Blood. 1998;92:32-39.

28. Cowland JB, Borregaard N. Molecular character ization and pattern of tissue expression of the gene for neutrophil gelatinase-associated lipocalin from humans. Genomics. 1997;45:17-23.

29. Chono H, Yoshioka H, Ueno M, Kato I. Removal of inhibitory substances with recombinant fibronectin- $\mathrm{CH}-296$ plates enhances the retroviral transduction efficiency of CD34(+)CD38(-) bone marrow cells. J Biochem (Tokyo). 2001;130:331334.

30. de Wet JR, Wood KV, DeLuca M, Helinski DR, Subramani S. Firefly luciferase gene: structure and expression in mammalian cells. Mol Cell Biol. 1987;7:725-737

31. van der Eb AJ, Graham FL. Assay of transforming activity of tumor virus DNA. Methods Enzymol. 1980;65:826-839.

32. Barge RM, de Koning JP, Pouwels K, et al. Tryptophan 650 of human granulocyte colony-stimulating factor (G-CSF) receptor, implicated in the activation of JAK2, is also required for G-CSFmediated activation of signaling complexes of the p21ras route. Blood. 1996;87:2148-2153.

33. Lowenberg B, van Putten WL, Touw IP, Delwel R, Santini V. Autonomous proliferation of leukemic cells in vitro as a determinant of prognosis in adult acute myeloid leukemia. N Engl J Med. 1993;328:614-619.

34. Valk PJ, Vankan Y, Joosten M, et al. Retroviral insertions in Evi12, a novel common virus integration site upstream of Tra1/Grp94, frequently coincide with insertions in the gene encoding the peripheral cannabinoid receptor Cnr2. J Virol. 1999; 73:3595-3602.

35. Ward AC, Smith L, de Koning JP, van Aesch $Y$, Touw IP. Multiple signals mediate proliferation, differentiation, and survival from the granulocyte colony-stimulating factor receptor in myeloid 32D cells. J Biol Chem. 1999;274:14956-14962.

36. Yang WM, Yao YL, Seto E. The FK506-binding protein 25 functionally associates with histone deacetylases and with transcription factor YY1. EMBO J. 2001;20:4814-4825.

37. Garcia E, Marcos-Gutierrez C, del Mar LM, Moreno JC, Vidal M. RYBP, a new repressor protein that interacts with components of the mammalian Polycomb complex, and with the transcription factor YY1. EMBO J. 1999;18:3404-3418.

38. David G, Alland L, Hong SH, et al. Histone deacetylase associated with $\mathrm{mSin} 3 \mathrm{~A}$ mediates repression by the acute promyelocytic leukemiaassociated PLZF protein. Oncogene. 1998;16: 2549-2556.

39. Wu WS, Vallian S, Seto E, et al. The growth suppressor PML represses transcription by functionally and physically interacting with histone deacetylases. Mol Cell Biol. 2001;21:2259-2268.

40. Gelmetti V, Zhang J, Fanelli M, et al. Aberrant re cruitment of the nuclear receptor corepressorhistone deacetylase complex by the acute myeloid leukemia fusion partner ETO. Mol Cell Biol. 1998;18:7185-7191.

41. Amann JM, Nip J, Strom DK, et al. ETO, a target of $t(8 ; 21)$ in acute leukemia, makes distinct contacts with multiple histone deacetylases and binds $m S i n 3 A$ through its oligomerization domain. Mol Cell Biol. 2001;21:6470-6483.

42. Shrivastava A, Saleque S, Kalpana GV, et al. Inhibition of transcriptional regulator Yin-Yang-1 by association with c-Myc. Science. 1993;262:18891892.

43. Shrivastava A, Yu J, Artandi S, Calame K. YY1 and c-Myc associate in vivo in a manner that depends on c-Myc levels. Proc Natl Acad Sci U S A. 1996;93:10638-10641.

44. Mori A, Higashi H, Hoshikawa Y, et al. Granulocytic differentiation of myeloid progenitor cells by $\mathrm{p} 130$, the retinoblastoma tumor suppressor homologue. Oncogene. 1999;18:6209-6221. 